\title{
Accesibilidad a tratamiento en personas con problemas de Alcohol y otras Sustancias de abuso
}

\section{Access to treatment for people with alcohol and other substance abuse problems}

\author{
GAIL GILCHRIST*, ${ }^{* \star}$; Francina FonseCA ${ }^{\star},{ }^{* \star *}$; \\ MARTA TORRENS ${ }^{\star},{ }^{\star \star \star},{ }^{\star \star \star \star \star}$
}

* Grup de Recerca en Trastorns per Us de Substàncies. Programa de Neuropsicofarmacologia. Institut de Recerca Hospital del Mar (IMIM). Barcelona

** School of Health and Social Care, University of Greenwich. London

*** Institut de Neuropsiquiatria i Addiccions (INAD) - Parc de Salut Mar, Barcelona

**** Departament de Psiquiatria, Universitat Autònoma de Barcelona. Barcelona

\section{RESUMEN}

Objetivos: El estudio IATPAD (Estudio para la Mejora del Acceso a Tratamiento en personas con problemas de Alcohol y otras Drogas), es un estudio europeo cuyo objetivo es detectar barreras y facilitadores al acceso a tratamiento de pacientes con trastornos relacionados con alcohol y drogas. En este artículo se presentan los resultados del estudio cuantitativo en Cataluña.

Metodología: Estudio multicéntrico, transversal. Se evaluaron los tres puntos principales de entrada a tratamiento: Psiquiatría General (CSMA), Centros de Atención a Adicciones (CAS) y Centros de Atención Primaria (CAP). La muestra de centros fue seleccionada de forma aleatoria. Se utilizó la Escala de Condición Medica (ECM) para evaluar la consideración del personal hacia pacientes con problemas de alcohol y drogas.

Resultados: Se detectaron diferencias según el tipo de centro en la consideración hacia pacientes con problemas de alcohol, drogas y depresión; los profesionales de los CAS presentaban mayor consideración hacia los pacientes adictos que los profesionales de CSMA $(p<0,001)$ o CAP $(p<0,001)$.

Conclusiones: Las actitudes de los profesionales hacia los pacientes con trastorno por uso de sustancias son importantes a la hora de conseguir una vinculación de estos pacientes y mejorarlas debe ser tenido en cuenta en las politicas sobre tratamiento.

Palabras clave: Actitudes del personal de la salud, alcoholismo/tratamiento, medicina de familia, psiquiatría general, trastornos por usos de sustancias/tratamiento

\section{ABSTRACT}

Objectives: IATPAD (Improvement in Access to Treatment for People with Alcohol and Drug Related Problems) is a European study. The study's main objective was to detect barriers and facilitators associated with access to treatment among patients with problems related to alcohol and drug use. This article presents the quantitative findings from Cataluña (Spain).

Methods: Multicentric, cross-sectional study. Three main treatment entry points were assessed: General Psychiatry (CSMA), Out-patient Addiction Centers (CAS) and Primary Care (CAP). Centres were randomly selected. The Medical Condition Regard Scale was used to measure regard for working with different patient groups (diabetes, depression, patients with problems related to alcohol and patients with problems related to drugs). Results: Differences were detected in regard towards working with patients with alcohol, drugs and depression by treatment entry point. Professionals from the CASs reported higher regard towards working with substance users than professionals from CSMAs $(p<0,001)$ or professionals from CAPs $(p<0,001)$.

Conclusions: The attitudes of professionals towards patients with substance use disorders are important for treatment engagement. Treatment policy needs to consider how to improve staff attitudes.

Key words: Alcoholism/therapy, attitude of health personnel, physicians, primary health care psychiatrists, substance-related disorders/therapy. 


\section{INTRODUCCIÓN}

L os trastornos adictivos son enfermedades crónicas y con tendencia a la recidiva con importantes repercusiones personales, familiares y sociales, que pueden tratarse de forma eficaz ${ }^{1}$. Por ello es importante que las personas con problemas por consumo de sustancias puedan acceder a los servicios de tratamiento y rehabilitación disponibles y asequibles en un intervalo de tiempo razonable. En este sentido es importante minimizar las barreras que puedan dificultar la accesibilidad al tratamiento. Estas barreras pueden ser muy variadas. Algunas provienen del propio paciente, como la falta de motivación, la creencia que uno mismo puede manejar el problema sin necesidad de tratamiento, o que los tratamientos que se ofrecen son de escasa calidad y no son efectivos. Otras barreras provienen de la organización asistencial: complejidad en los mecanismos de admisión a tratamiento de estos pacientes, escasa empatía por esta patología, saturación de los dispositivos asistenciales, desconocimiento de los servicios de tratamiento y dificultades de accesibilidad geográficas ${ }^{2-7}$. Y finalmente las actitudes negativas de los trabajadores de la salud respecto de los trastornos adictivos $^{8-18}$ pueden influir no sólo en la calidad de la atención ${ }^{16,}$ 17, 19 sino también dificultar el acceso al tratamiento de estos pacientes ${ }^{20}$.

Por otra parte, los factores que se han relacionado con un mejor acceso a tratamiento son: la necesidad de tratamiento percibida por parte del paciente, la gravedad del problema, ${ }^{7,21}$ el funcionamiento social ${ }^{4}$ y el apoyo social para la abstinencia ${ }^{22}$.

Con el objetivo de detectar de las barreras que limitan y los factores que facilitan el acceso al tratamiento de los pacientes con problemas relacionados con el consumo de alcohol y otras drogas, en distintos países europeos, se llevó a cabo el proyecto IATPAD (Estudio para la Mejora del Acceso a Tratamiento en personas con problemas de Alcohol y otras Drogas) ${ }^{23}$. En este artículo describimos los principales hallazgos del estudio cuantitativo realizado en Cataluña ${ }^{23}$.

\section{MÉTODOS}

\section{Diseño del estudio}

Se trata de un estudio comparativo y transversal en el cual se evalúan los sistemas de tratamiento y se valoran las actitudes hacia el trabajo con pacientes con dependencia de alcohol y otras sustancias.

\section{Evaluación de los sistemas de tratamiento:}

En Cataluña, disponemos de tres redes de atención general a los pacientes: la red de salud general, en la que el principal dispositivo comunitario es el Centro de Atención Primaria (CAP), la red de salud mental, en la que el dispositivo comunitario es el Centro de Salud Mental de Adultos (CSMA), y la red específica de atención a las drogodepen- dencias, en los que se atendienden directamente los pacientes con problemas por consumo de alcohol y otras drogas, principalmente en los Centros de Atención y Seguimiento de las Drogodependencias (CAS).

Se evaluó la accesibilidad a tratamiento de los tres puntos de entrada principales en Catalunya: CAP, CSMA y CAS, mediante un formulario sobre el centro que incluia: descripción de la cartera de servicios, los perfiles de pacientes y profesionales, las listas de espera y el horario de atención a los pacientes y que debía ser contestado por el coordinador del centro.

Para ello se seleccionaron de forma aleatoria una muestra del 30\% (20/66) de todos los CSMA en Cataluña estratificados en función de la densidad de población por $\mathrm{km}^{2} \mathrm{y}$ se incluyeron tres centros adicionales para asegurar que la muestra era representativa de todos los centros y regiones sanitarias de Cataluña. Si el CSMA seleccionado aleatoriamente, aceptaba participar en el estudio, se invitó al CAP y CAS de la misma área de influencia a participar en el estudio.

\section{Encuesta de la Escala de Condición Médica (ECM):}

A una muestra de conveniencia de 187 profesionales (médicos, psiquiatras, psicólogos, enfermeros, trabajadores sociales,...) de los centros seleccionados en la fase previa se les solicitó que completaran la ECM. Cada miembro del personal facilitó su consentimiento informado antes de participar en el estudio. La ECM es una escala de actitudes referentes al trabajo con pacientes con problemas relacionados con el consumo de alcohol y drogas, depresión y diabetes ${ }^{24}$.

La ECM es un instrumento válido y fiable que consta de 11 ítems para valorar los sesgos, las emociones y las expectativas generadas por la consideración hacia una determinada patología. La puntuación máxima es de 66 (máxima consideración) y la mínima de 11 (menor consideración). La escala fue traducida al español y la retrotraducción de la versión española fue aprobada por los autores. Dado que la ECM no había sido validada previamente en todos los grupos profesionales que fueron incluidos en este estudio, los investigadores realizaron un análisis factorial para determinar qué dimensiones se podrían identificar con los 11 ítems. Se revelaron 3 factores en relación a los pacientes con problemas relacionados con el consumo de sustancias: 1) actitud negativa (rechazo) en los ítems $3,5,6,9,11 ; 2$ ) actitud positiva en los ítems $1,4,9,10$ y 3 ) actitud neutra (sin diferencias entre un tipo de paciente y otro: ítems $2,6^{23}$.

\section{Análisis}

Para todos los análisis se utilizó el software estadistico SPSS. Se calcularon las puntuaciones totales, la media y desviación estándar (DE) para cada una de las cuatro condiciones en la ECM. Se calcularon las diferencias en la consideración hacia pacientes con diferentes patologías según sexo, edad, tiempo trabajando en la profesión, tipo de profesión y 
tipo de centro mediante pruebas de t y ANOVA con análisis post-hoc (Scheffe). Para analizar las posibles asociaciones entre las puntuaciones en las diferentes patologías según el profesional, se realizó una prueba de ANOVA multifactor.

\section{RESULTADOS}

\section{Evaluación de los sistemas de tratamiento}

Se reclutaron un total de 57 servicios: 15 CAP, 20 CSMA y 22 CAS. La tasa de respuesta de los centros fue del $82,6 \%$ (57/69). La tasa de respuesta por centros fue: en CAP de $65 \%(15 / 23)$, la de los CSMA de $87 \%(20 / 23)$ y la de los CAS del 96\% (22/23). Seis centros se negaron a participar y seis no respondieron y no fue posible contactar. En la Tabla 1 se describen las potenciales barreras y facilitadores de acceso al tratamiento. Se observaron diferencias significativas en las horas de atención entre los centros. Los CAP ofrecian un horario más amplio con más días de obertura que los CSMA $(p=0,016)$ y los CAS $(p=0,005)$ y también abrian durante más horas (CAP vs. CSMA; $p<0.001$; CAP vs. CAS; $p<0.001)$. En general, el 80\% (40/50) de los centros refirieron que había una lista de espera para su servicio. Se detectaron diferencias en función del tipo de centro en relación a la lista de espera: 100\% (17/17) de los CSMA, el 90\% $(18 / 20)$ de los CAS, y 38,5\% (5/13) de los CAP ( $p<0,001)$. Para aquellos con lista de espera, el tiempo medio de espera para una cita osciló entre 1-60 días (media 20,1; DE: 15,6). Los CAP $(p<0,001)$ y los CAS $(p<0,001)$ tenían tiempos de espera para una primera cita significativamente inferiores que los CSMA. Cuatro $(4 / 18 ; 22,2 \%)$ de los CSMA y ningún CAP declaró que su servicio estaba especializado en problemas de alcohol y/o drogas.

\section{Encuesta de la ECM}

De los 187 profesionales que completaron la ECM, 47 eran médicos generales, 42 psicólogos, 42 enfermeros, 31 trabajadores sociales y 25 psiquiatras. La mayoria eran mujeres $(130 / 187 ; 69,5 \%)$ y trabajando un promedio de 15,8 años (de 9,0$)$.

La consideración de los profesionales hacia los pacientes con problemas de alcohol (media: 48,63; IC 95\%: 47,3449,92 ) y otras drogas (media: 46,93; IC 95\%: 45,48-48,39) fue significativamente menor que para los pacientes con depresión (media: 53,31; IC 95\%: 52,31-54,30) o diabetes (media: 52,27; IC 95\%: 51,20-53,34) $(p<0,05)$.

Las profesionales femeninas presentaron menor consideración hacia los pacientes con problemas de alcohol que los profesionales maculinos (Tabla 2). No hubo diferencias en las puntuaciones ECM por edad, región sanitaria de los centros, ni por el tiempo que habían estado trabajando en su profesión.

Se detectaron diferencias según el tipo de centro en la consideración hacia pacientes con problemas de alcohol, pacientes con problemas de drogas y los pacientes con depresión (Tabla 3). Los profesionales procedentes de los CAS tenían mayor consideración hacia los pacientes con

Tabla 1. Descripción de las barreras y facilitadores potenciales según tipo de centro

\begin{tabular}{|c|c|c|c|c|}
\hline & $\begin{array}{c}\text { CSMA } \\
\mathrm{N}=20^{\wedge} \\
\text { Media (DE) }\end{array}$ & $\begin{array}{c}\text { CAS } \\
\mathrm{N}=22^{\wedge} \\
\text { Media (DE) }\end{array}$ & $\begin{array}{c}\text { CAP } \\
\mathrm{N}=15^{\wedge} \\
\text { Media (DE) }\end{array}$ & $\mathrm{p}$ \\
\hline Número de dias abierto cada semana & $5,0(0,0)$ & $4,9(1,2)$ & $5,9(0,8)$ & 0,003 \\
\hline Número de horas abierto cada semana & $42,1(10,5)$ & $39,6(13,1)$ & $93,7(44,6)$ & $<0,001$ \\
\hline Horas abierto en dias laborables & $42,1(10,5)$ & $38,8(12,4)$ & $75,4(25,6)$ & $<0,001$ \\
\hline Horas abierto los fines de semana & $0(-)$ & $0,8(1,9)$ & $18,3(20,9)$ & $<0,001$ \\
\hline Tiempo medio de espera para la primera cita (dias) & $32,5(14,4)$ & $14,5(9,1)$ & $1,9(0,9)$ & $<0,001$ \\
\hline Tiempo medio de espera para tratamiento desde la primera cita (dias) & $8,2(12,1)$ & $3,0(4,8)$ & $6,5(6,4)$ & 0,319 \\
\hline & $N(\%)$ & N (\%) & N (\%) & \\
\hline Acuden personas con problemas de alcohol/drogas & $10(83,3)$ & $20(100)$ & $11(100)$ & 0,067 \\
\hline Servicio especializado en problemas de alcohol/drogas & $4(22,2)$ & $20(100)$ & $0(-)$ & $<0,001$ \\
\hline $\begin{array}{l}\text { Pacientes tienen que demostrar que tienen seguridad social/ seguro de enfermedad para conseguir } \\
\text { acceso a la atención }\end{array}$ & $11(68,8)$ & $12(60,0)$ & $9(81,8)$ & 0,458 \\
\hline
\end{tabular}

¿ Discrepancias en totales debido a preguntas no contestadas

CSMA: Centro de Salud Mental Adultos; CAS: Centro de Atención y Seguimiento a las Adicciones; CAP: Centro de Atención Primaria; DE: desviación estándar 
Tabla 2. Puntuación del MCRS según el sexo del profesional

\begin{tabular}{|c|c|c|c|c|c|}
\hline Condición de MCRS & $\mathrm{N}$ & Sexo & Media & (DE) & $\mathrm{p}$ \\
\hline \multirow[t]{2}{*}{ Alcohol } & 57 & Hombre & 50,59 & $(7,86)$ & 0,046 \\
\hline & 129 & Mujer & 47,76 & $(9,25)$ & \\
\hline \multirow[t]{2}{*}{ Drogas } & 56 & Hombre & 48,50 & $(8,51)$ & 0,161 \\
\hline & 127 & Mujer & 46,25 & $(10,53)$ & \\
\hline \multirow[t]{2}{*}{ Depresión } & 55 & Hombre & 54,71 & $(5,96)$ & 0,065 \\
\hline & 125 & Mujer & 52,69 & $(7,04)$ & \\
\hline \multirow[t]{2}{*}{ Diabetes } & 52 & Hombre & 53,25 & $(6,90)$ & 0,230 \\
\hline & 116 & Mujer & 51,83 & $(7,09)$ & \\
\hline
\end{tabular}

DE: desviación estándar
Tabla 3. Puntuación del MCRS según condición y centros

\begin{tabular}{l|ccccc}
\hline Condición de MCRS & N & Centros & Media & $($ DE $)$ & $p$ \\
\hline Alcohol & 64 & CSMA & 46,71 & $(7,60)$ & $<0,001$ \\
& 59 & CAS & 54,20 & $(6,66)$ & \\
& 52 & CAP & 44,39 & $(9,48)$ & \\
& 11 & CSMA/CAS & 49,91 & $(9,55)$ & \\
\hline \multirow{2}{*}{ Drogas } & 61 & CSMA & 44,47 & $(8,42)$ & $<0,001$ \\
& 56 & CAS & 53,42 & $(7,81)$ & \\
& 52 & CAP & 42,36 & $(10,25)$ & \\
& 11 & CSMA/CAS & 47,45 & $(10,32)$ & \\
\hline \multirow{2}{*}{ Depresión } & 61 & CSMA & 55,18 & $(5,60)$ & 0,003 \\
& 59 & CAS & 53,96 & $(6,06)$ & \\
& 52 & CAP & 50,54 & $(8,09)$ & \\
& 11 & CSMA/ CAS & 52,64 & $(5,84)$ & \\
\hline \multirow{2}{*}{ Diabetes } & 56 & CSMA & 51,81 & $(6,70)$ & 0,350 \\
& 49 & CAS & 51,69 & $(7,51)$ & \\
& 52 & CAP & 53,68 & $(6,89)$ & \\
& 11 & CSMA/ CAS & 50,55 & $(7,17)$ & \\
& & & & & \\
& & & & & \\
& & &
\end{tabular}

CSMA: Centro de Salud Mental Adultos; CAS: Centro de Atención y Seguimiento a las Adicciones; CAP: Centro de Atención Primaria; DE: desviación estándar

Tabla 4. MCRS según condición y tipo de profesional

\begin{tabular}{|c|c|c|c|c|c|}
\hline Condición de MCRS & $\mathrm{n}$ & Profesión & Media & (DE) & $\mathrm{p}$ \\
\hline \multirow[t]{5}{*}{ Alcohol } & 47 & Médico & 47,33 & $(9,03)$ & 0,115 \\
\hline & 42 & Psicólogo* & 51,80 & $(8,01)$ & \\
\hline & 25 & Psiquiatra & 48,88 & $(9,86)$ & \\
\hline & 30 & Trabajador social & 47,36 & $(7,27)$ & \\
\hline & 42 & Enfermera & 47,67 & $(9,74)$ & \\
\hline \multirow[t]{5}{*}{ Drogas } & 47 & Médico & 45,48 & $(10,15)$ & 0,143 \\
\hline & 42 & Psicólogo* & 50,07 & $(9,28)$ & \\
\hline & 24 & Psiquiatra & 47,62 & $(10,10)$ & \\
\hline & 29 & Trabajador social & 44,63 & $(10,28)$ & \\
\hline & 41 & Enfermera & 46,62 & $(9,87)$ & \\
\hline \multirow[t]{5}{*}{ Depresión } & 47 & Médico & 52,99 & $(7,37)$ & 0,001 \\
\hline & 42 & Psicólogo* & 55,60 & $(4,72)$ & \\
\hline & 24 & Psiquiatra & 56,20 & $(7,26)$ & \\
\hline & 27 & Trabajador social & 49,88 & $(5,99)$ & \\
\hline & 40 & Enfermera & 51,85 & $(6,86)$ & \\
\hline \multirow[t]{5}{*}{ Diabetes } & 47 & Médico & 53,88 & $(6,18)$ & 0,049 \\
\hline & 36 & Psicólogo* & 51,27 & $(7,04)$ & \\
\hline & 22 & Psiquiatra & 51,90 & $(9,14)$ & \\
\hline & 24 & Trabajador social & 49,04 & $(5,56)$ & \\
\hline & 39 & Enfermera & 53,45 & $(6,99)$ & \\
\hline
\end{tabular}

* Incluye a un psicoterapeuta familiar y un educador social

DE: desviación estándar

problemas de alcohol y los pacientes con problemas de drogas que los profesionales de los CSMA $(p<0,001)$ o los CAP $(p<0,001)$. Los profesionales de los CSMA tenian mayor consideración hacia los pacientes con problemas de alcohol ( $p$ $<0,001)$ y los pacientes con depresión $(p=0,004)$ que los profesionales de los CAP (análisis post hoc de Scheffe no mostrado).

En el análisis ANOVA multifactor, se observaron diferencias en cuanto a los pacientes con depresión y los pacientes con diabetes fueron encontradas según el tipo de profesión (Tabla 4). Los psiquiatras $(p=0,020)$ y psicólogos $(p=0,015)$ mostraban mayor consideración que los trabajadores sociales para los pacientes con depresión (análisis Post hoc Scheffe no mostrado).

\section{DISCUSIÓN}

En este estudio hemos descrito como la consideración de los profesionales era menor hacia los pacientes con trastorno por uso de sustancias (especialmente drogas diferentes del alcohol) comparado con los pacientes con depresión o diabetes. Estos resultados eran especialmente evidentes en el 
grupo de atención primaria. Estos resultados son consistentes con otros estudios en los que se describe como los médicos generales consideran que su formación no es adecuada para detectar y tratar este tipo de pacientes ${ }^{12,25}$ o bien que no disponen de suficiente tiempo para atenderlos de forma adecuada $^{26 .}$ Otros estudios similares destacan la opinión que estos pacientes deben ser atendidos en centros especializados ${ }^{27}$. Hay que destacar también la importancia de la motivación personal para acceder a tratamiento. Diferentes estudios han demostrado como mediante técnicas de entrevista motivacional se mejora la vinculación en servicios de addicciones ${ }^{28,29}$. El aprendizaje por parte de los profesionales de las diferentes "puertas de entrada" de técnicas motivacionales y su aplicación (estrategias económicas, por otro lado), permiten mejorar la vinculación de pacientes a tratamiento.

Hay que destacar también los resultados en relación a la consideracion hacia los pacientes con trastorno depresivo, que también es más baja que hacia diabetes en los profesionales de los centros de atención primaria. Estos resultados no son únicos de nuestro país sino que se han descrito también en otros estudios y reflejan la persistencia de la estigmatización de la enfermedad mental así como la falta de formación de muchos profesionales en su abordaje $\mathrm{e}^{30,31}$.

Cuando analizamos diferencias en cuanto al género, observamos que para el alcohol, las profesionales mujeres, presentan una menor consideración hacia estos pacientes. En la literatura ha sido escasamente estudiado el efecto del sexo del profesional en la relación médico-paciente. Se han descrito diferencias en la práctica entre médicos hombres y mujeres; en una revisión reciente ${ }^{32}$ se describe como las profesionales mujeres ofrecen más servicios preventivos y consejo, mientras que los hombres dedican más tiempo a recoger la historia clínica y la exploración física.

En cuanto a las puntuaciones por profesional, destacar también la menor consideración del grupo de trabajo social hacia los pacientes con depresión y diabetes, que puede ser un reflejo del menor conocimiento de estas patologías.

Mejorar las actitudes relacionadas con los consumidores de sustancias mediante campañas en los medios hará que a muchos pacientes con un trastorno por dependencia de sustancias, les resulte mucho más fácil acudir a los servicios y es posible que se reduzca el tiempo que transcurre desde el reconocimiento del problema hasta la entrada en el tratamiento.

La educación de la población, pero también de grupos profesionales seleccionados, cumple una importante función en el proceso que facilita el acceso a información adecuada del grupo diana. Debería prestarse una atención especial a los familiares de las personas en riesgo, o a aquellas que tienen familiares con problemas de alcoholismo o drogadicción.

La principal limitación de este estudio radica en que el instrumento utilizado en la fase cuantitativa (EMC) fue previamente diseñado y utilizado en estudiantes de medicina y enfermería, por lo que su aplicabilidad en otras profesiones no había sido validada.
Destacan como puntos fuertes de este estudio el ser el primer estudio que compara las actitudes de los diferentes profesionales a trabajar con pacientes con trastornos adictivos comparado con pacientes con otras enfermedades crónicas; de la misma forma, tampoco tenemos conocimiento de otros estudios que comparen actitudes de diferentes profesionales en diferentes puntos de entrada al tratamiento. El tamaño muestral y la randomización de los centros en Catalunya permiten generalizar nuestros resultados.

Las principales implicaciones para el tratamiento tienen que ver con las actitudes negativas de los profesionales y mejorarlas en todos aquellas personas que trabajen con pacientes con problemas de adicciones. Una revisión reciente muestra como la cultura de una determianda organización puede tener un importante impacto en las actitudes de los profesionales hacia los pacientes con problemas por uso de sustancias, y cómo el ambiente puede influir en cambios en la práctica tras recibir formación ${ }^{33}$. De todas formas, no sólo la formación de los trabajadores es necesaria, ya que las actitudes también están relacionadas con el entorno cultural y los recursos institucionales.

\section{AGRADECIMIENTOS}

Los autores declaran que no existe ningún conflicto de interés. El estudio ha sido financiado por la European Commission Directorate of Public Health and Risk Assessment (contract number 2005322), por el Departament de Salut de la Generalitat de Catalunya y por la Red de Trastornos Adictivos (RTA-RD06/0001/1009).

Los autores quieren agradecer a los pacientes y al personal de los los Centros de Salud Mental de Adultos, los Centros de Atención y Seguimiento de Drogodependencias y los Centros de Atención Primaria por su participación en el proyecto. Queremos también agradecer al resto de paises europeos implicados en el estudio IATPAD.

\section{REFERENCIAS}

1. Sellman D. The 10 most important things known about addiction. Addiction 2010; 105: 6-13.

2. Alegria M, Perez DJ, Williams $S$. The role of public policies in reducing mental health status disparities for people of color. Health Aff (Millwood) 2003; 22: 51-64.

3. Festinger DS, Lamb RJ, Kountz MR, Kirby KC, Marlowe D. Pretreatment dropout as a function of treatment delay and client variables. Addict Behav 1995; 20: 111-5.

4. Hser $\mathrm{YI}$, Maglione M, Polinsky ML, Anglin MD. Predicting drug treatment entry among treatment-seeking individuals. J Subst Abuse Treat 1998; 15: 213-220.

5. Mowbray 0, Perron BE, Bohnert AS, Krentzman AR, Vaughn MG. Service use and barriers to care among heroin users: results from a national survey. Am J Drug Alcohol Abuse 2010; 36: 305310. 
6. Myers B, Fakier N, Louw J. Stigma, treatment beliefs, and substance abuse treatment use in historically disadvantaged communities. Afr J Psychiatry (Johannesbg) 2009; 12: 218-222.

7. Tucker JA, Vuchinich RE, Rippens PD. A factor analytic study of influences on patterns of help-seeking among treated and untreated alcohol dependent persons. J Subst Abuse Treat 2004; 26: 237-242.

8. Ding $L$, Landon BE, Wilson IB, Wong MD, Shapiro MF, Cleary PD. Predictors and consequences of negative physician attitudes toward HIV-infected injection drug users. Arch Intern Med 2005; 165: 618-623.

9. Furlan PM, Laguzzi S, Rosa M, Barboni S, Roagna G, Scarso G. Attitudes, knowledge and methods of assistance used by general practitioners in Novara in treating drug addicts. Study of 100 doctors. Minerva Psichiatr 1990; 31: 145-149.

10. Lindberg M, Vergara C, Wild-Wesley R, Gruman C. Physicians-intraining attitudes toward caring for and working with patients with alcohol and drug abuse diagnoses. South Med J 2006; 99: 28-35.

11. Roche AM, Guray C, Saunders JB. General practitioners' experiences of patients with drug and alcohol problems. $\mathrm{Br} J$ Addict 1991; 86: 263-275.

12. Tantam D, Donmall M, Webster A, Strang J. Do general practitioners and general psychiatrists want to look after drug misusers? Evaluation of a non-specialist treatment policy. Br J Gen Pract 1993; 43: 470-474.

13. Todd FC, Sellman JD, Robertson PJ. Barriers to optimal care for patients with coexisting substance use and mental health disorders. Aust N Z J Psychiatry 2002; 36: 792-799.

14. Sheridan J, Strang J, Barber N, Glanz A. Role of community pharmacies in relation to HIV prevention and drug misuse: findings from the 1995 national survey in England and Wales. BMJ 1996; 313: 272-274.

15. Howard MO, Chung SS. Nurses' attitudes toward substance misusers. I. Surveys. Subst Use Misuse 2000; 35: 347-365.

16. Carroll J. Attitudes of professionals to drug abusers. Br J Nurs 1993; 2: 705-711.

17. Deehan A, Taylor C, Strang J. The general practitioner, the drug misuser, and the alcohol misuser: major differences in general practitioner activity, therapeutic commitment, and 'shared care' proposals. Br J Gen Pract 1997; 47: 705-709.

18. Digiusto $E_{1}$ Treloar C. Equity of access to treatment, and barriers to treatment for illicit drug use in Australia. Addiction 2007; 102: 958-969.

19. Baldacchino A, Gilchrist G, Fleming R, Bannister J. Guilty until proven innocent: a qualitative study of the management of chronic non-cancer pain among patients with a history of substance abuse. Addict Behav 2010; 35: 270-272.

20. Neale J, Tompkins C, Sheard L. Barriers to accessing generic health and social care services: a qualitative study of injecting drug users. Health Soc Care Community 2008; 16: 147-154.

21. Simpson CA, Tucker JA. Temporal sequencing of alcohol-related problems, problem recognition, and help-seeking episodes. Addict Behav 2002; 27: 659-674.

22. Brown BS, O'Grady K, Battjes RJ, Farrell EV. Factors associated with treatment outcomes in an aftercare population. Am J Addict 2004; 13: 447-460.

23. Gilchrist G, Moskalewicz J, Slezakova S, Okruhlica L, Torrens $M$, Vajd $R$, et al. Staff regard towards working with substance users: a European multi-centre study. Addiction 2011; 106: 1114-1125.

24. Christison GW, Haviland MG, Riggs ML. The medical condition regard scale: measuring reactions to diagnoses. Acad Med 2002; 77: 257-262.

25. McGillion J, Wanigaratne S, Feinmann C, Godden T, Byrne A. GPs' attitudes towards the treatment of drug misusers. Br J Gen Pract 2000; 50: 385-386.

26. Davies A, Huxley P. Survey of general practitioners' opinions on treatment of opiate users. BMJ 1997; 314: 1173-1174.

27. Matheson C, Pitcairn J, Bond CM, van Teijlingen E, Ryan M. General practice management of illicit drug users in Scotland: a national survey. Addiction 2003; 98: 119-126.

28. Carroll KM, Ball SA, Nich C, Martino S, Frankforter TL, Farentinos $C_{\text {, et }}$ al. Motivational interviewing to improve treatment engagement and outcome in individuals seeking treatment for substance abuse: a multisite effectiveness study. Drug Alcohol Depend 2006; 81: 301-312.

29. Carroll KM, Libby B, Sheehan J, Hyland N. Motivational interviewing to enhance treatment initiation in substance abusers: an effectiveness study. Am J Addict 2001; 10: 335-339.

30. Richards JC, Ryan P, McCabe MP, Groom G, Hickie IB. Barriers to the effective management of depression in general practice. Aust N Z J Psychiatry 2004; 38: 795-803

31. Dowrick C, Gask L, Perry R, Dixon C, Usherwood T. Do general practitioners' attitudes towards depression predict their clinical behaviour? Psychol Med 2000; 30: 413-419.

32. Bertakis KD. The influence of gender on the doctor-patient interaction. Patient Educ Couns 2009; 76: 356-360.

33. Pidd K, Freeman T, Skinner N, Addy D, Shoobridge J, Roche AM. From Training to Work Practice Change: An Examination of Factors Influencing Training Transfer in the Alcohol and Other Drugs Field 2004. 